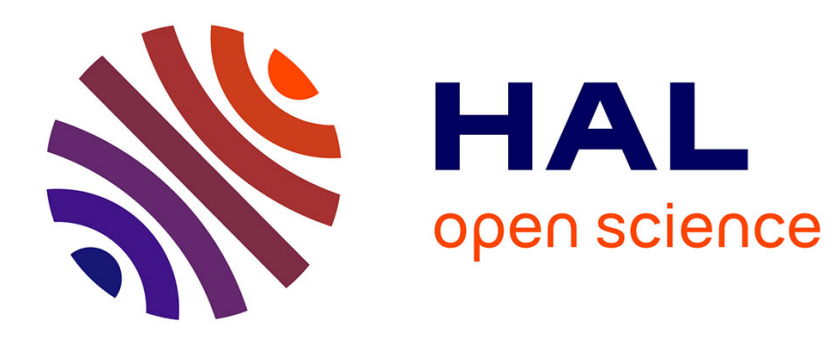

\title{
Modelling of three-body effects in a double continuum
}

R. Tweed, C. Tannous, P. Marchalant

\section{To cite this version:}

R. Tweed, C. Tannous, P. Marchalant. Modelling of three-body effects in a double continuum. Journal de Physique IV Proceedings, 1993, 03 (C6), pp.C6-107-C6-116. 10.1051/jp4:1993610 . jpa-00251695

\section{HAL Id: jpa-00251695 https://hal.science/jpa-00251695}

Submitted on 1 Jan 1993

HAL is a multi-disciplinary open access archive for the deposit and dissemination of scientific research documents, whether they are published or not. The documents may come from teaching and research institutions in France or abroad, or from public or private research centers.
L'archive ouverte pluridisciplinaire HAL, est destinée au dépôt et à la diffusion de documents scientifiques de niveau recherche, publiés ou non, émanant des établissements d'enseignement et de recherche français ou étrangers, des laboratoires publics ou privés. 


\title{
Modelling of three-body effects in a double continuum
}

\author{
R.J. TWEED, C. TANNOUS and P. MARCHALANT
}

Laboratoire de Collisions Electroniques et Atomiques, Equipe ERS003 du CNRS, Faculté des Sciences et Techniques, 6 avenue le Gorgeu, BP. 452, 29275 Brest cedex, France

\begin{abstract}
Theoretical calculations of double ionisation by electron or photon impact require a final state wavefunction which takes account both of the coulomb repulsion between the pair of free electrons and of their interaction with the residual ionic core. It is desirable that this should be separable so as to facilitate the introduction of electron-pair correlations in the initial state wavefunction for the collision system. We propose a method for calculating coupled classical trajectories for the free electrons and deducing from these potentials from which their quantum mechanical wavefunctions may be obtained. Test calculations are reported for electron impact single ionisation of hydrogen.
\end{abstract}

\section{INTRODUCTION}

Two major problems are encountered when making calculations for (e,2e) processes. One is how to take account of the coulomb repulsion between a pair of final state free electrons. This also exists in the case of double ionisation by photon impact $(\gamma, 2 e)$ or by high energy electron impact $(e, 3 e)$. It is resolved by explicit introduction of a coulomb correlation factor in the wavefunction of Brauner, Briggs and Klar [1] and in subsequent developments by Altick and collaborators [2]. The other difficulty is that of coupling between competing reaction channels, which also results from coulomb forces between electron pairs. This must obviously be taken into account when obtaining the final state of the target system in the cases of ionisation-withexcitation and of autoionisation. A similar problem exists for the complete collision wavefunction describing the projectile and the target system together. Curran and walters [3] deal with it by using close-coupling wavefunctions, describing the elastic and two of the excitation channels exactly and other channels through the use of pseudostates. Joachain and collaborators [4] employ Born 2 or Eikonal Born series models including some channels explicitly and compensating for the rest by a closure approximation. Distorted wave Born models [5] only take account of the elastic channel. Because the Brauner et al wavefunction is an assymptotic solution of the full three-body coulomb Hamiltonian it implicitly contains some coupling to elastic and excitation channels. Pseudostates or closure can introduce 
approximate coupling to double continuum channels. All these models can give good results in appropriate circumstances, but none fully includes the physics of the problem.

The Brauner et al wavefunction, although a considerable advance, is strictly correct only in the assymptotic region. It provides a good target final state for double ionisation of helium by photons [6] or electrons [7]. But its extension to more complex targets is delicate. The problem of introducing the interaction of two free electrons with a many-electron ionic core can in principle be solved by replacing the Coulomb waves for the individual electrons by wavefunctions calculated in the static field of the core. But the coulomb correlation term (involving the relative directions of the two free electrons) is not, without further approximation, easily expressed in partial wave form. Nevertheless, it is inevitable that the target initial state and the ionic core be described by multiconfiguration Hartree-Fock type wavefunctions. Formulation of the collision problem, without the use of partial wave expansions and the support of Racah algebra, then becomes arduous.

In high energy electron impact, both for double ionisation and for ionisation-with-excitation, the target initial and final states must be described by wavefunctions taking proper account of electron-pair correlations. Plane waves may be used to describe the projectile. At lower energies it is certainly necessary to take account of the coupling between competing reaction channels. A possible approach would be to combine the representation of the complete collisional system by a close-coupling wavefunction and an approximate description of the final state, including electron-pair correlations for the target system. This is envisagable only if the final state wavefunction is simple enough. In previous work [8] we proposed a parametric potential method for treating coupled channels based on different excited states of the residual ion. Here we examine a classical trajectory method for dealing with the double continuum.

\section{COULOMB CORRELATIONS IN THE DOUBLE CONTINUUM}

As an alternative to introducing an explicit coulomb correlation factor, we wish to develop a model in which the wavefunction for the double continuum is separable. Each electron is to be described by a distorted wave, calculated using a potential obtained from classical trajectories and specific to particular kinematics (energies and relative angle). Such an approach was adopted by Jones el al [9] and by Robaux et al [10], using constant speed linear trajectories; as compared to a standard Distorted Wave Born (D.W.B.A.) model this implies modifying the potentials used for the calculation of the final state free electron wavefunctions. For $e^{-}-$He collisions this implies adding a term with $\lambda / r$ behaviour to standard distortion potentials; such a procedure gives fairly good cross sections. But for $e^{-}-H$ collisions it comes down to using a product of coulomb waves with an effective charge prescription similar to that of Schultz [11] and the results are unsatisfactory. Very recently, Jones et al [12] have developed a D.W.B.A. model in which a correlation factor, related to the Brauner-Briggs-Klar (B.B.K.) wavefunction, is introduced in the final state. This apparently gives excellent results for $e^{-}-H$ collisions. Whelan et al [13] have applied a correlation correction to a standard D.W.B.A. model and get very good shape comparison with the experiments of Rösel et al [14] for $e^{-}$- He collisions. We note that the B.B.K. theory can have problems for angular distributions at low incident electron energy in $e^{e}-\mathrm{H}$ collisions under coplanar symmetric energy-sharing conditions [15] and neither the B.B.K. nor the D.W.B.A 
theories give a reliable absolute value for cross sections below about $100 \mathrm{eV}$. The sucess of the theories of Jones et al and of whelan et al results from the fact that they combine the B.B.K. and D.W.B.A. approaches. The model described in the present paper is an alternative approach to theirs, with the same object.

We have developed methods for the calculation of coupled classical trajectories, taking account of their curvature and of the change of energy due to the coulomb repulsive force. The system of two electrons moving in the field of a nucleus can become chaotic if their trajectories are taken out to infinite distance [16]. Thus we integrate to a finite but very large radius (at least $8000 \mathrm{a}_{0}$ ). Furthermore, the trajectories have closest approach distances related to the energetics in a particular kinematical situation. This forces us to make an analytical continuation of our perturbation potentials down to zero radius, avoiding singular behaviour at the origin. This is one of the difficulties in our method, but is essentially a numerical one because the perturbation is insignificant compared to the nuclear attraction at small radius. A much more serious problem is the conceptual one related to the wavelength associated with the energy of the colliding electron. For slow electrons this is very much larger than the dimensions of the target, so one could naively suppose that a classical treatment has no sense. However, when the colliding electron approaches the nucleus, it is accelerated by falling into a deep attractive potential well and so its wavelength decreases. The adiabatic approximation used to derive polarisation potentials fails at small radius (when the colliding electron penetrates well inside the target electron cloud) precisely because of this acceleration.

Consider the general problem of two free electrons interacting with eachother in the field of an ion, represented by the central potential V. Their coordinates are $r_{1}$ and $r_{2}$ and their separation is $r=r_{2}-r_{1}$. They are detected at angles $\theta_{a}$ and $-\theta_{b}$, with energies $E_{a}$ and $E_{b}$ respectively. We consider their motion to be confined to the plane defined by the ion and the detectors, so $\mathbf{r}_{i}=\left\{\boldsymbol{r}_{\mathbf{i}}, \theta_{i}\right\}$ and $\mathbf{r}=\{\mathbf{r}, \theta\}$ with $\theta=\theta_{2}-\theta_{1}$. We calculate their classical trajectories as a function of time, taking into account the effect of their mutual repulsion on their individual energies $E_{i}(t)$ and angular momenta $1_{i}(t)$. For electron 1 we have:

$$
\begin{aligned}
& T_{i}(t)=\frac{m}{2}\left(\left(\frac{d r_{1}}{d t}\right)^{2}+r_{1}^{2}\left(\frac{d \theta_{1}}{d t}\right)^{2}\right)=E_{1}\left(r_{1}\right)-V\left(r_{1}\right) \\
& I_{1}(t)=m r_{1}^{2}\left(\frac{d \theta_{1}}{d t}\right)^{2}=I_{1}(0)-\int_{0}^{t} \frac{r_{1}\left(t^{\prime}\right) r_{2}\left(t^{\prime}\right) \sin \theta\left(t^{\prime}\right)}{r^{3}\left(t^{\prime}\right)} d t^{\prime} \\
& E_{1}(t)=E_{1}(0)+\int_{0}^{t} \frac{1}{r^{3}\left(t^{\prime}\right)}\left\{\left[r_{1}\left(t^{\prime}\right)-r_{2}\left(t^{\prime}\right) \cos \theta\left(t^{\prime}\right)\right] \frac{d r_{1}}{d t^{\prime}}\right. \\
& \left.-r_{1}\left(t^{\prime}\right) r_{2}\left(t^{\prime}\right) \sin \theta\left(t^{\prime}\right) \frac{d \theta_{1}}{d t^{\prime}}\right\} d t^{\prime}
\end{aligned}
$$

A similar set of equations for electron 2 are obtained by permuting the indexes 1 and 2 and changing the sign before $\sin \theta$. The factors in the integrals come from the radial and angular components of the repulsive force. The $T_{i}(t)$ are the kinetic energies on the trajectories, so the effective potential for the motion of electron $i$ is simply $V_{j}(t)=E_{j}(\infty)-T_{i}(t)$. This gives us the potential for quantum mechanical calculation of its wavefunction. 
It is obviously impossible to define initial conditions for $\mathbf{r}_{i}(0)$, $l_{j}(0)$ and $E_{j}(0)$ in a non-arbitrary way. However, the derivation in quantum mechanics of scattering amplitudes, for single ionisation by Peterkop [17] and by Rudge and Seaton [18], and for double ionisation by Tweed [19], imply an assymptotic condition on the momenta $k_{a}, k_{b}$ and $\mathbf{k}=\mathbf{k}_{\mathrm{b}}-\mathbf{k}_{\mathrm{a}}$ :

$$
\begin{array}{lll}
r_{1}=\tau k_{a} & r_{2}=\tau k_{b} & r=\tau k
\end{array}
$$

where $\tau$ is a constant. Imposing this condition in the calculation of our classical trajectories, we obtain from equation (2):

$l_{1}(t) \underset{t \rightarrow \infty}{\sim}-\frac{k_{a} k_{b} \sin \left(\theta_{a}+\theta_{b}\right)}{v k^{2}} \ln (r)+$ constant

At sufficiently large radius and time, $r_{1} d \theta_{1} / d t$ becomes small in comparison with $d r_{1} / d t$. So from equation (3) we obtain:

$E_{1}(t) \underset{t \rightarrow \infty}{\sim}-\frac{k_{a}-k_{b} \cos \left(\theta_{a}+\theta_{b}\right)}{k} r_{1}^{-1}+$ constant

Equation (4) corresponds to the result obtained by Gailitis [20]: the coulomb repulsion between electrons causes their individual angular momenta to increase logarithmically with radius, but the total angular momentum of the electron pair is conserved and is finite. The choice of constant is arbitrary; for the results presented here it was taken to be zero. In equation (5) the constant is $E_{a}$, so as to get the required energy at the detector, assuming this is at sufficiently large radius for the interaction potentials to be negligible.

We use the assymptotic forms from equations (4) and (5) to provide initial estimates for $l_{j}$ and $E_{i}$. These are injected into equations (1) and (2), which are then solved simultaneously for $\mathbf{r}_{i}$ and $\theta_{i}$ by numerical integration. The initial values of $\theta_{1}$ and $\theta_{2}$ are adjusted so as to get the required assymptotic angles, $\theta_{a}$ and $-\theta_{b}$ respectively. Those of $r_{1}$ and $r_{2}$ are essentially closest approach distances imposed by the repulsive force between the electron pair becoming too strong. The $l_{i}$ and $E_{i}$ are then recalculated from the trajectories, with initial values chosen so as to get the required assymptotic result. The procedure is then continued iteratively; convergence is rapid.

From these trajectory calculations we obtain the $v_{i}(t)$ and $r_{i}(t)$ on the same time grid. Thus we can express $v_{i}$ as a function of $r_{i}$ on any radial grid by means of cubic spline interpolation. We finally obtain a potential $v_{a}\left(r_{1}\right)$ for the electron which is detected at angle $\theta_{a}$ with energy $E_{a}$ and a potential $V_{b}\left(r_{2}\right)$ for the electron which is detected at angle $-\theta_{b}$ with energy $E_{b}$.

\section{ELECTRON-IMPACT SINGLE IONISATION OF HYDROGEN}

We have used electron impact single ionisation of hydrogen as a test case. The triple differential cross section is given by:

$\frac{d^{3} \sigma}{d \Omega_{a} d \Omega_{b} d E}=(2 \pi)^{4} \frac{k_{a} k_{b}}{k_{0}} \frac{1}{4} \sum_{S}(2 S+1)\left|f_{s}\left(k_{a}, k_{b}\right)\right|^{2}$

with 


$$
\begin{aligned}
f_{s}\left(k_{a}, k_{b}\right)=\int d r_{1} \int d r_{2} \Psi_{s}^{\star}\left(r_{1}, r_{2}\right)\left\{1 / r-1 / r_{2}-v_{s}\left(r_{2}\right)\right\} \\
\times\left[\Phi_{a}\left(r_{1}\right) \Phi_{b}\left(r_{2}\right)+(-1)^{s} \Phi_{a}\left(r_{2}\right) \Phi_{b}\left(r_{1}\right)\right]
\end{aligned}
$$

where $\Phi_{a}$ and $\Phi_{b}$ are distorted waves calculated using potentials $V_{a}$ and $V_{b}$ corresponding to the particular final momenta $k_{a}$ and $k_{b}: \Psi_{s}$ describes the collisional system for total spin $s$, with an incident electron of momentum $\mathbf{k}_{0}$. It is obtained by the distorted wave polarised orbital method of McDowell et al [21] and takes into account, through the distortion potential $v_{s}$, of elastic scattering of the colliding electron. For the sake of comparison, we also make calculations using the same initial state $\Psi_{s}$ but with coulomb waves for $\Phi_{a}$ and $\Phi_{b}$. These correspond essentially a standard distorted wave Born model. We refer to them as "DWB" in figure legends whereas the calculations with classical potentials are refered to as "DWB+PCI".

Cross sections are plotted as a function of $\theta_{b}$ for incident energy $E_{0}$ $=54.4 \mathrm{eV}$ in figure 1 , for $\mathrm{E}_{\mathrm{b}}=5 \mathrm{eV}$ and $\theta_{\mathrm{a}}=4^{\circ}$. We compare the results of our two calculations to the experimental data of schlemmer et al [22] as normalised by Brauner et al [15]. The classical potentials give a good result, seemingly overestimating slightly the magnitude of the binary lobe.

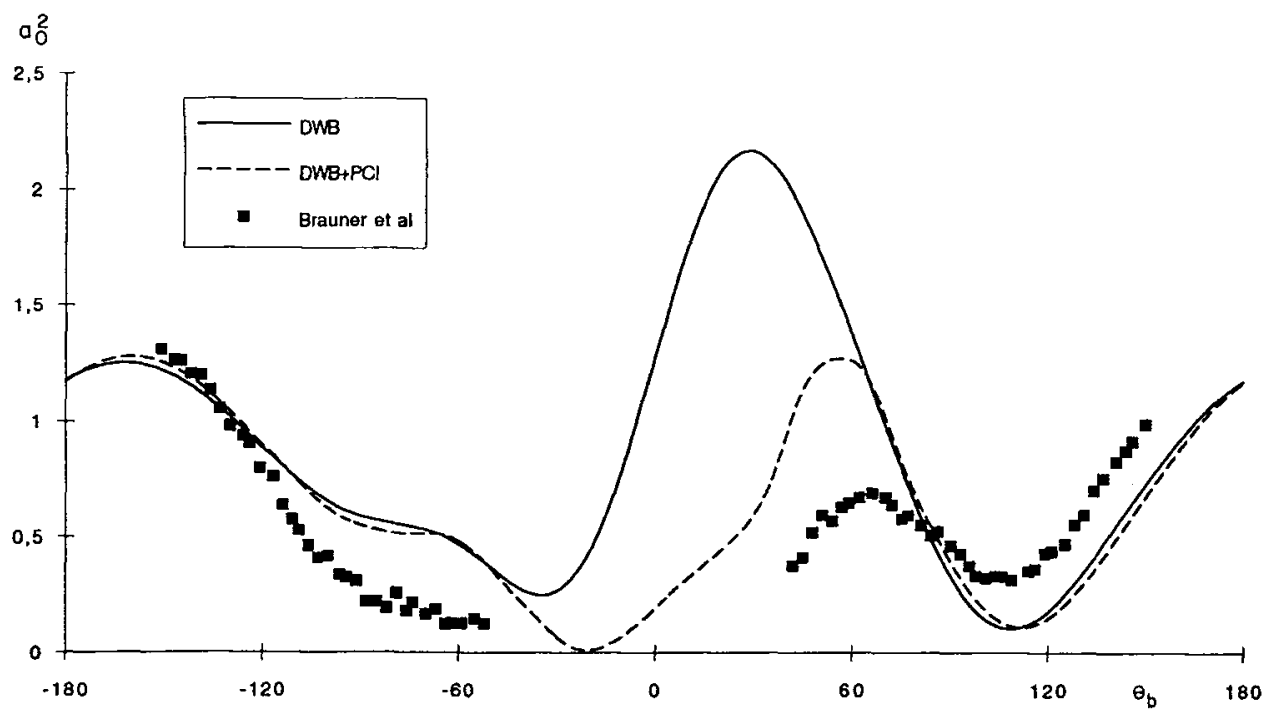

Figure 1: T.D.C.S in $a_{0}^{2} / \mathrm{sr}^{2} / 2 \mathrm{Ry}$ for $E_{0}=54.4 \mathrm{eV}, \mathrm{E}_{\mathrm{b}}=5 \mathrm{eV}$ and $\theta_{\mathrm{a}}=4^{\circ}$

In figure 2 we compare our two calculations to those of curran and walters [3]. The experimental points are as in figure 1, but now normalised to the latter calcultion. This gives a different picture: our classical potential calculations give a good description of the binary lobe but a poor description of the recoil lobe. A similar comparison is made in figure 3 for the same energies and $\theta_{0}=10^{\circ}$. since the calculations of curran and walters take account of the coupling between excitation channels they correctly describe the recoil lobe, whereas our $\Psi_{s}$ is too simple an approximation. However, as they have no post-collision interaction in their final state, we do better than them for the binary lobe. 


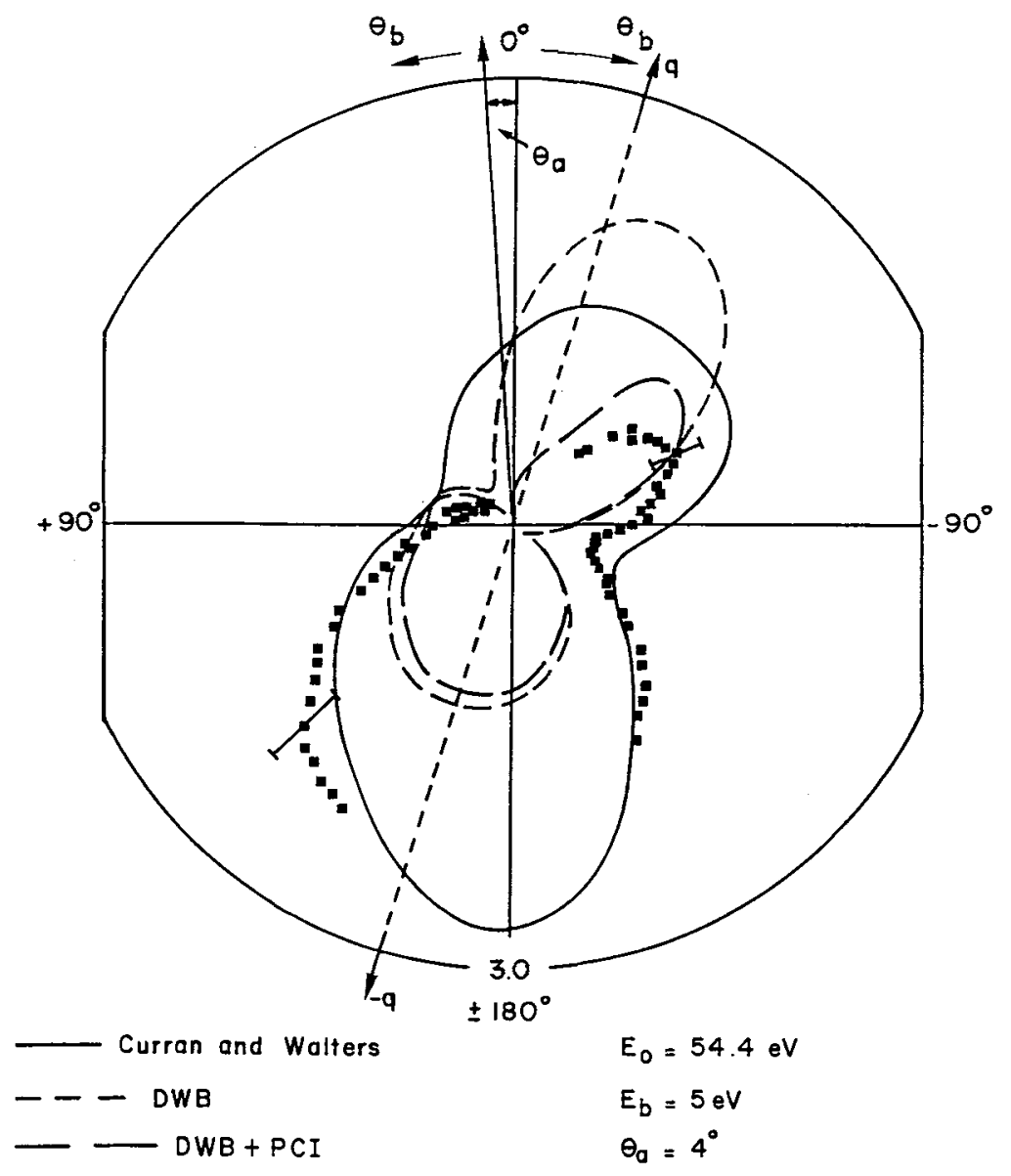

Figure 2: As figure 1, with an alternative nomalisation of experiment

It is of extreme interest that Jones et al [12] are able to reproduce both the binary and the recoil lobe of the experiments at $\theta_{a}=4^{\circ} \mathrm{With}$ a normalisation factor like that of figure 2 here. We in fact get essentially the same result as them for the binary lobe. This is an indication that their correlation factor is bringing in something more than just a description of post collision interactions. In the same way that a good initial state wavefunction can in a certain sense predict the future, a good final state wavefunction can remember the past. our final state wavefunction is constructed to introduce a specifically post-collision effect. So if we wish to improve our model we must work on pre-collision phenomena by improving the description of the coupling between the elastic, excitation and ionisation channels. Our initial state wavefunction describes the elastic channel quite well and introduces some coupling to inelastic channels through a long-range polarisation potential. What we must now introduce are short range correlations related to penetration of the target charge-cloud by the colliding electron. Maybe we will not gain in the end in simplicity, but we will have the advantage of being able to dissociate pre- and post-collision mechanisms. The work of whelan et al [13] has a similar aim. 


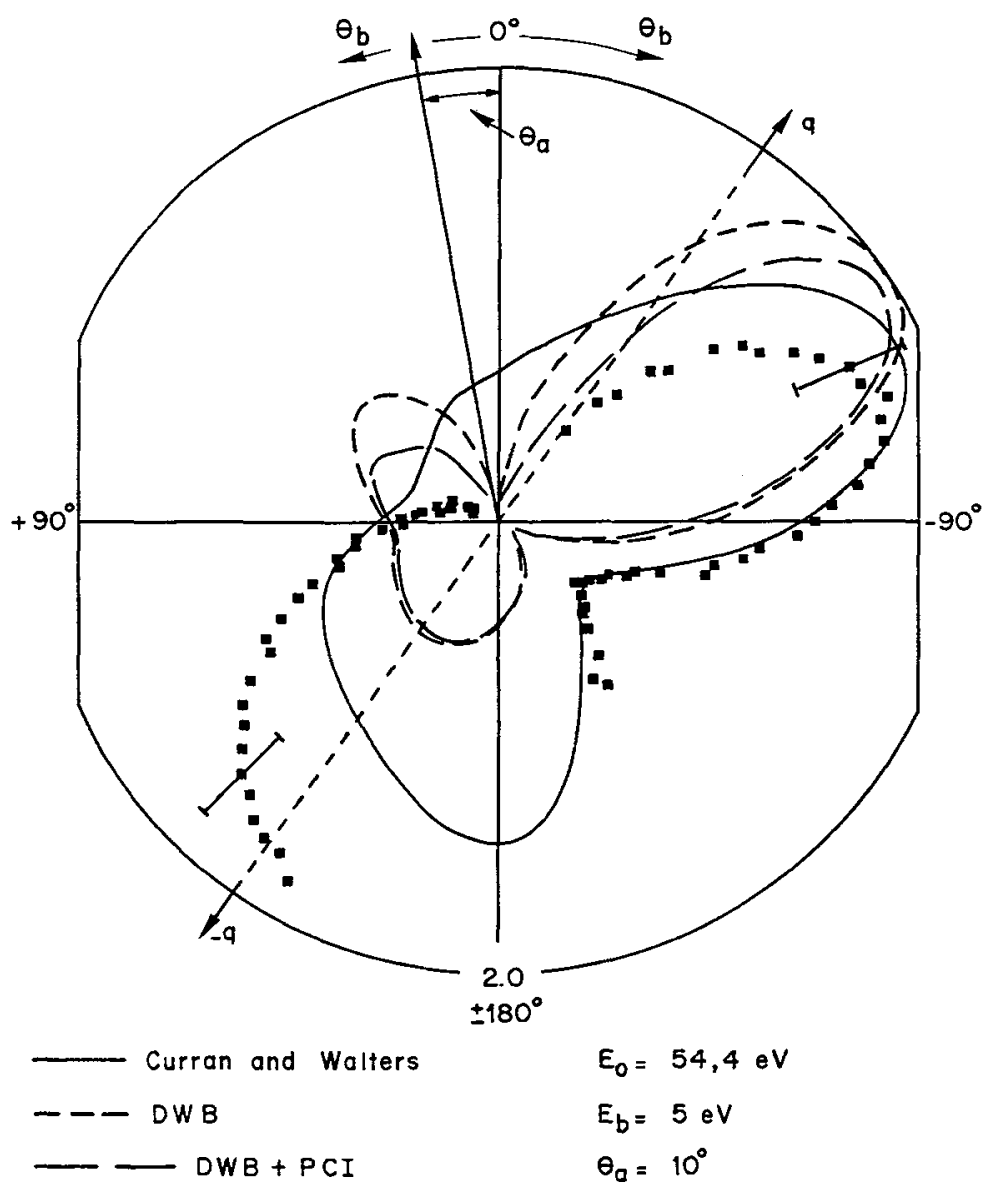

Figure 3: T.D.C.S in $\mathrm{a}_{0}^{2} / \mathrm{sr}^{2} / 2 \mathrm{RY}$ for $\mathrm{E}_{0}=54.4 \mathrm{eV}, \mathrm{E}_{\mathrm{b}}=5 \mathrm{eV}$ and $\theta_{\mathrm{a}}=10^{\circ}$

It is also interesting to consider the case of an incident energy of 27.2 eV with $\mathrm{E}_{\mathrm{a}}=\mathrm{E}_{\mathrm{b}}=6.8 \mathrm{eV}$. We compare our two calculations to the data of Brauner et al [15], plotting the cross section as a function of $\theta_{a}+\theta_{b}$, for $\theta_{a}=15^{\circ}$ in figure 4 and for $\theta_{a}=30^{\circ}$ in figure 5 . Our results are acceptable except for the fact that the cross section does not drop to zero for a relative angle of $0^{\circ}$. This is not suprising when $\Phi_{a}^{\prime \prime}$ and $\Phi_{\mathrm{b}}$ are Coulomb waves, but the introduction of a post-collision interaction should prevent two electrons of the same energy from having the same direction. We are able to reproduce the double peak form of the experiment because our initial state $\Psi_{0}$ gives a good description of the scattering of the projectile by the nucleus. However, the peaks are not in the right place. Our classical potentials are not giving a strong enough effect at small relative angle.

The problem is the choice of a zero constant in the assymptotic condition for the angular momentum (equation 4). As a result, the angular momenta at small radius are not large enough to permit a rapid separation of the electrons and the trajectories have to be started too far out. We are now testing a computer code using inward integration of the coupled classical equations of motion in Cartesian coodinates $\left\{x_{i}, y_{i}\right\}$. Boundary conditions on derivatives $\mathrm{dx}_{\mathrm{i}} / \mathrm{dt}$ and 
$d y_{i} / d t$ at large time are affected only slightly by the $\ln (r)$ factor in equation 4 , and negligibly by the constant.

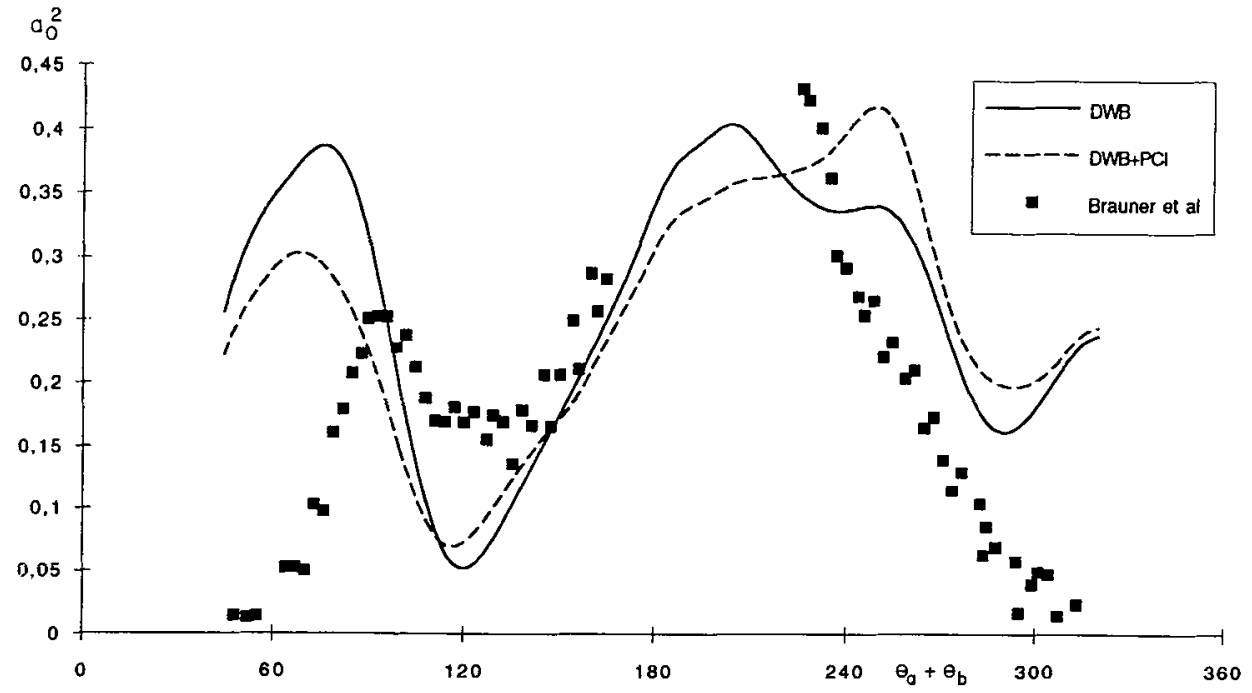

Figure 4: T.D.C.S in $a_{0}^{2} / \mathrm{sr}^{2} / 2 \mathrm{RY}$ for $E_{0}=27.2 \mathrm{eV}, E_{a}=E_{b}=6.8 \mathrm{eV}$ and $\theta_{a}=15^{\circ}$ as a function of $\theta_{a}+\theta_{b}$.

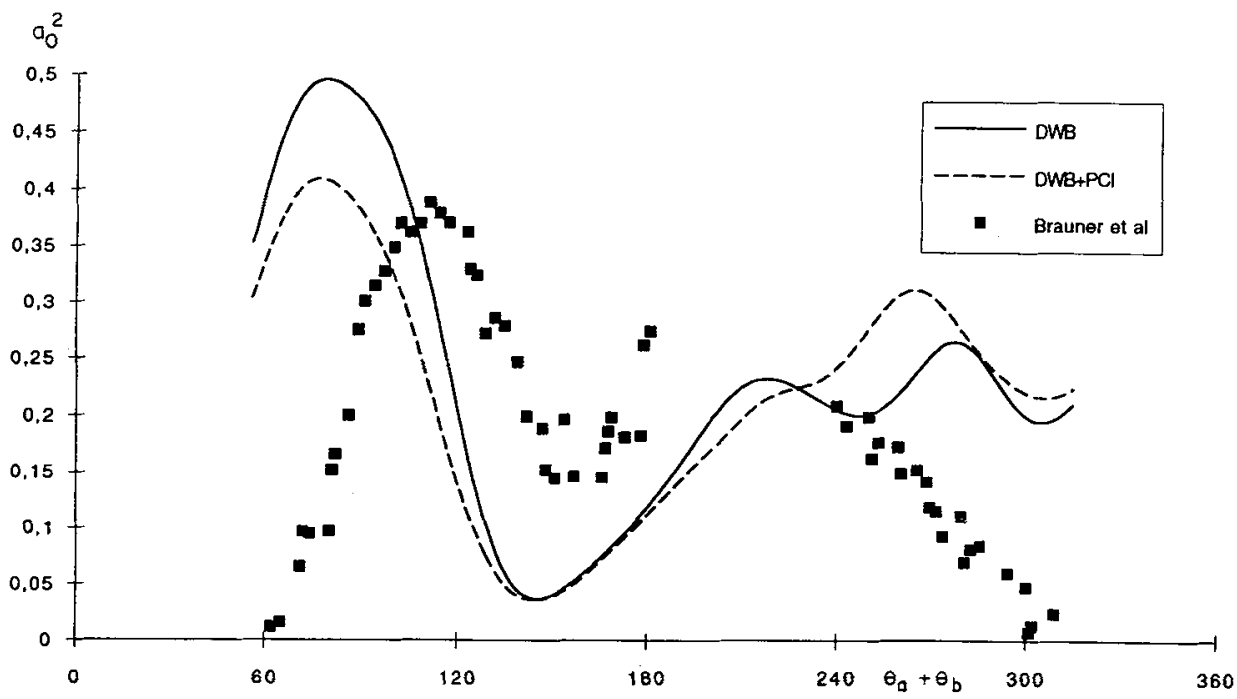

Figure 5: As figure 4 for $\theta_{a}=30^{\circ}$; experimental data $\times 2$.

In figure 6 we show some typical results of these computations for the case of two free electrons of energy $6.8 \mathrm{ev}$. We plot the $\mathrm{r}$-multiplied correction to the central residual ion potential: $r_{1}\left(V_{a}\left(r_{1}\right)-V\left(r_{1}\right)\right)$. It may be seen that as the angle between the directions of the electrons decreses, the potential becomes increasingly important at small to medium radius. This is precisely what we wish to obtain, 


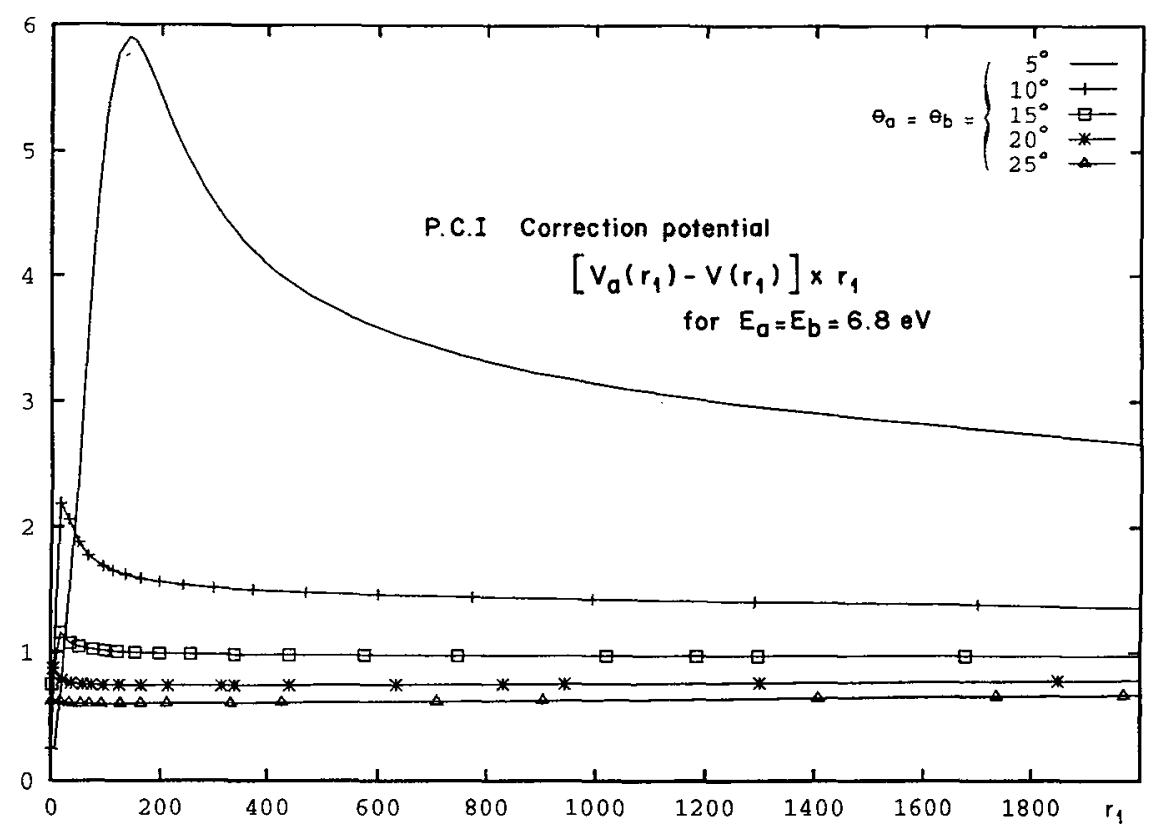

Figure 6: Dependence on relative angle of the classical correction potentials, for two electrons of energy $6.8 \mathrm{eV}$

since the high peak in the potential at small radius will push the wavefunctions of the final state free electrons out to larger radius. This will reduce their overlap with the wavefunction of the bound target electron in the initial state and so give smaller radial integrals. The larger is the peak in the potential, the smaller will be the cross section. So at very small relative angles of the electrons we should obtain a near-zero cross section.

\section{CONCLUSIONS}

our present method shows promise but needs improvement, especially when the energies of the two free electrons are both small. The problem comes from the correct definition of assymptotic boundary conditions, and in current work we are systematically extending the final time and radii out until we can be sure of the uniqueness of the classical solutions. Use of Cartesian coordinates simplifies the numerical aspects and makes it possible to bring the trajectories in to a very small radius. So we should be able to introduce the coulomb repulsion between electrons at short distance. Extention to manyelectron systems is simple. There remains the problem of short range correlations. This affects somewhat the potentials which we derive from our classical trajectories, but it seems probable that the real problem is with the wavefunction of the colliding electron in the initial state. We are currently introducing potentials obtained by the method of Perdew and zunger [23] and Bachelet et al [24].

\section{ACKNOWLEDGMENTS}

Professors J. Langlois and $O$. Robaux contributed useful ideas and discussions. D. Rozuel helped prepare the figures. C. Tannous wishes to thank the Brest collisions group for their hospitality. 


\section{REFERENCES}

[1] Brauner M. Briggs J.S. and Klar H., J. Phys. B 22 (1989) 2265

[2] Franz A. and Altick P.L., J. Phys. B 25 (1992) 1577

Ming Tong and Altick P.L., J. Phys. B 25 (1992) 741

[3] Curran E.P. and Walters H.R.J., J. Phys. B 20 (1987) 337

[4] Byron F.W. Jr., Joachain C.J. and Piraux B., J. Phys. B 13 (1980) L673

Potvliege R.M., Vucic S. and Joachain C.J., J.Phys. B 20 (1987) 4883

[5] McCarthy I.E., Z. Phys. D 4 (1992) 287

[6] Maulbetsch F. and Briggs J.S., J. Phys. B 26 (1993) 1679

[7] Joulakien B., Dal Cappello C. and Brauner M., J. Phys. B 25 (1992) 2863

[8] Robaux 0., Tweed R.J. and Langlois J., J. Phys. B 24 (1991) 4567

[9] Jones S., Madison D.H. and Srivastava K.M., J. Phys. B 25 (1992) 1899

[10] Robaux O., Tweed R.J. and Langlois J., Z. Phys. D 23 (1992) 331

[11] Schulz M., J. Phys. B 6 (1973) 2580

[12] Jones S., Madison D.H., Franz A. and Altick P.L., Phys. Rev. A 48 (1993) R22

[13] Whelan C.T., Allan R.J., walters H.R.J. and zhang X. in " $(e, 2 e)$ \& related processes" ed. C.T. Whelan, H.R.J. Walters, A. LahmamBennani and H. Ehrhardt (Kluwer Academic Publishers, 1993)

[14] Rösel T., Dupré C., Roder J., Duguet A., Jung K., Lahmam-Bennani A. and Ehrhardt H., J. Phys. B 24 (1991) 3059

[15] Brauner M., Briggs J.S., Klar H., Broad T., Rösel T., Jung K. and Ehrhardt H., J. Phys. B 24 (1991) 657

[16] Yan Gu and Jian-Min Yuan, Phys. Rev. A 47 (1993) R2442

[17] Peterkop R., opt. Spectros. 13 (1962) 87

[18] Rudge M.R.H. and Seaton M.J., ProC. R. SOC A 283 (1965) 262

[19] Tweed R.J., J. Phys. B 6 (1973) 259

[20] Gailitis M., J. Phys. B 23 (1990) 85

[21] MCDowell M.R.C., Morgan L. and Myerscough V.P., Comput. Phys. Commun. $7(1974) 38$

[22] Schlemmer P., Rösel T., Jung K. and Ehrhardt H., J. Phys. B 22 (1989) 2179

[23] Perdew J.P. and zunger A. Phys. Rev. B 23 (1981) 5048

[24] Bachelet G.B., Hamann D.R. and Schlüter M. Phys. Rev. B 26 (1982) 4199 